\title{
Nanoscale Probes in Ultrafast Transmission Electron Microscopy
}

Aycan Yurtsever*

* Centre Energie, Matériaux et Télécommunications, Institut National de la Recherche Scientifique (INRS), 1650 Boulevard Lionel-Boulet, Varennes, QC, J3X 1S2, Canada

Capturing ultrafast dynamics at the microscopic level with combined spatial and temporal resolutions requires probes that have sufficient electron counts, coherency and temporal length. Pulsed electrons in an ultrafast transmission electron microscope (UTEM) exhibit such properties, which allows us to capture dynamic events in real space, diffraction domain and energy-loss/gain spectroscopy [1]. In a UTEM, an event is initiated by a femtosecond optical pulse and the ensuing change in the specimen is imaged by pulsed electrons. Images are formed in a stroboscopic fashion [2], which enables superior signal-to-noise levels with combined spatial, temporal and spectral resolutions. Recently, $6 \mathrm{~nm}$ spatial, less than $1 \mathrm{ps}$ temporal and $1.4 \mathrm{eV}$ energy resolutions were demonstrated simultaneously in energy-filtered real-space imaging [3].

In this particular contribution, we emphasize the probe forming capability of UTEM. Traditionally, focused electron probes provided one of the most powerful means to access many material properties with rich analytical information. Scanning transmission electron microscopy and spectrum imaging are two examples whose fundamental component is an atomic scale probe. It follows that, the capability to focus pulsed electrons on a nanoscale area, by retaining their femto/picosecond temporal characteristic, would open new avenues in this newly emerging field.

In a UTEM, such probes are formed by using the condenser elements of the microscope. Fig. 1a, shows a frame of convergent beam electron diffraction (CBED) pattern from a nanoscale volume of a silicon crystal, acquired stroboscopically. The pattern is captured at negative time that is when electron pulse arrives on the specimen before the photon pulse. The diffraction image contains all the well-known features of continuous-beam CBED with similar signal-to-noise levels. With such high quality frames, it is possible to investigate one, or all, of these features for dynamic behavior.

The temporal evolution of two Kikuchi bands are shown in Fig. 1b. In the data, it is evident that one of the bands start to oscillate (shift its position) at time zero, before which it is stationary. The oscillation has a pulse-like profile with a period of $30 \mathrm{ps}$ and an envelope width of $140 \mathrm{ps}$. We attributed this behavior to a laser induced, propagating strain wave caused by inhomogeneous sample thickness [4].

Another study where ultrafast electron probes are utilized is displayed in Fig. 2. In this example, plasmonic fields of a nanoparticle are mapped by rastering the probe in the area of interest. At every probe position and time delay, an electron energy gain/loss spectrum is acquired. The real-space intensity distribution of an inelastic scattering peak is reconstructed across the particle for every time delay. Here, only time zero and +200 fs are shown. For details of this work see Ref. [5].

Currently, UTEM is the only approach that can simultaneously reach the sub-10 nm and sub-1 ps domains in real space. With further improvements to electron counts and coherencies, perhaps it will be possible to reach the atomic resolution with ultrafast times [6]. 


\section{References}

[1] A.H. Zewail \& J.M. Thomas, "4D Electron Microscopy", Imperial College Press, London

[2] In stroboscopic acquisition, many pulses (up to a million) are used to form an image, a diffraction pattern or a spectrum at a given time delay.

[3] A. Yurtsever, J. S. Baskin and A. H. Zewail, Nano Letters 12, 5027 (2012)

[4] A. Yurtsever, S. Schaefer \& A.H. Zewail, Nano Letters, 12, 3772 (2012).

[5] A. Yurtsever, R.M. van der Veen \& A.H. Zewail, Science, 335, 59 (2012).

[6] Experiments were conducted in the group of Prof. Ahmed Zewail at the California Institute of Technology. The original work was published in [4] and [5].
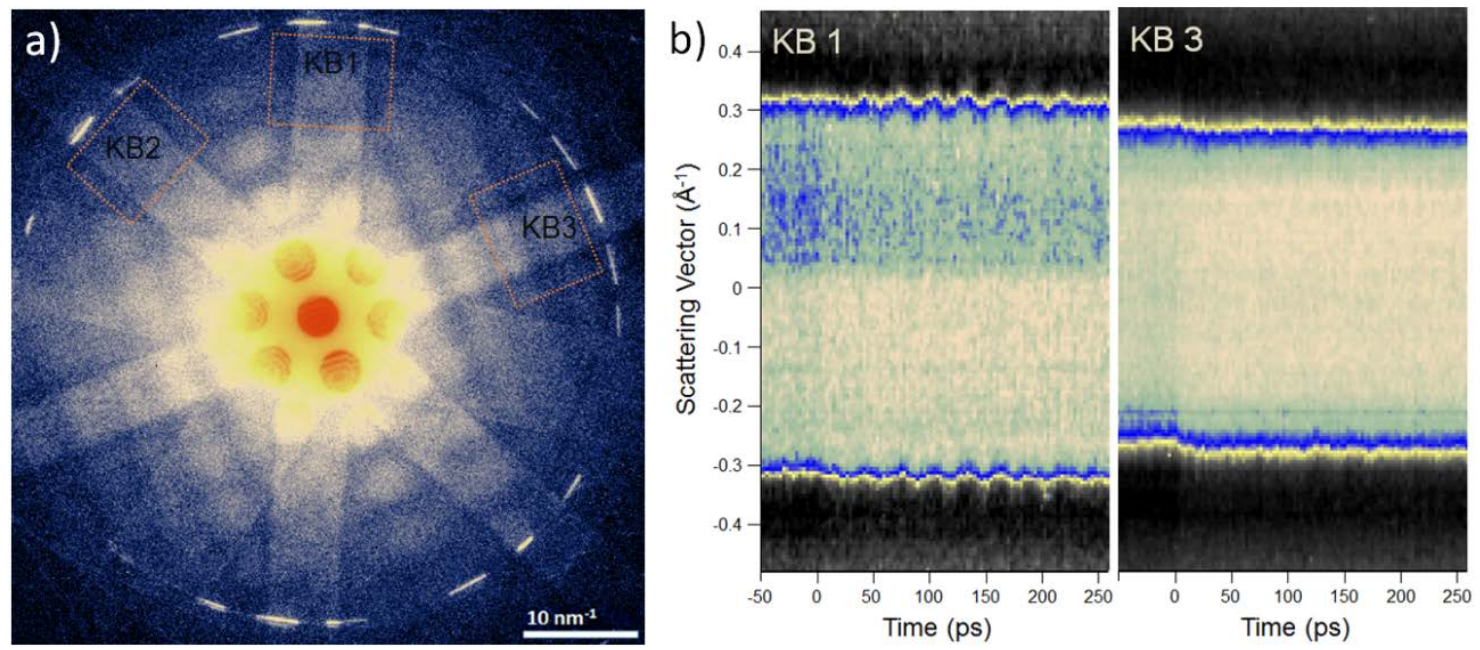

FIG. 1 a) A CBED frame taken at negative time, along the [-114] crystallographic direction of silicon. b) The temporal evolution of the two observed Kikuchi bands. The cross-section of each band is evaluated at the rectangular region indicated in a); they are plotted consecutively as a function of the time delay in b). The KB1 band shows strong oscillations (note the edges of the band), in the form of band shift, and exhibits a period of 30 ps; KB3 exhibits no oscillations. The sample was excited with green femtosecond laser pulse at $520 \mathrm{~nm}$ wavelength; repetition rate of the laser was $100 \mathrm{KHz}$.

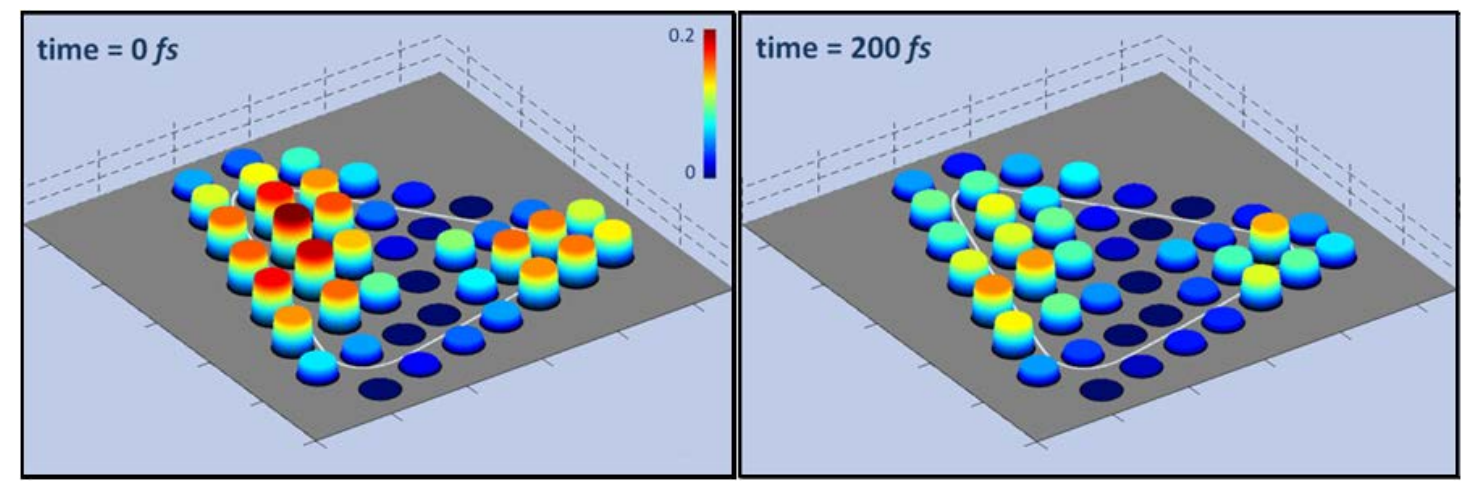

FIG. 2 Ultrafast nanoscale electron probes utilized for plasmonic near-field imaging. The plasmonic response of a triangular particle to the excitation laser is mapped in a way similar to spectrum imaging. Here are shown the strength of the fields at time zero and +200 fs. The full-with-at-halfmaxima of the temporal response is less than $1 \mathrm{ps}$ and the probe diameter is $10 \mathrm{~nm}$. 